\title{
Dobutamine Stress Echocardiography is Inadequate to Detect Early Cardiac Allograft Vasculopathy
}

\author{
Kevin J. Clerkin, MD, Maryjane A. Farr, MD, MSc, Susan W. Restaino, MD, Ziad A. Ali, MD, \\ DPhil, and Donna M. Mancini, MD \\ Department of Medicine, Division of Cardiology, Columbia University College of Physicians and \\ Surgeons, New York, New York, USA
}

\section{Introduction}

Cardiac allograft vasculopathy (CAV) is one of the major causes of morbidity and mortality following heart transplantation (HT) and is already present in $8 \%$ of patients by one year post-HT ${ }^{1}$. The end result is a pan-arterial vasculopathy leading to stenosis of coronary arteries and endothelial dysfunction of both the epicardial coronary arteries and the subendocardial microvasculature ${ }^{2}$. Dobutamine stress echocardiography (DSE) is commonly used to screen for CAV in patients with chronic kidney disease, and in some instances alternating with coronary angiography to minimize risk of progressive renal dysfunction. DSE carries a Class IIa recommendation from the ISHLT for CAV screening for those unable to undergo invasive evaluation ${ }^{3}$. Previous studies reported a good sensitivity (7295\%) and specificity (55-91\%) to detect luminal $\mathrm{CAV}^{4}$. A recent single center failed to replicate these findings among patients at least five years post-transplant, showing a sensitivity to detect CAV1-CAV3 of only $7-28 \%{ }^{5}$. We sought to determine the adequacy of DSE for CAV assessment in the first five years after transplant.

\section{Methods}

All DSE and angiograms of a prospectively followed cohort of 221 consecutive patients transplanted at Columbia University Medical Center (CUMC) from January $1^{\text {st }}, 2010-$ August $15^{\text {th }}, 2013$ were retrospectively reviewed through December $15^{\text {th }}, 2015$. Study of this patient cohort was approved by the CUMC Institutional Review Board. Institutional CAV screening protocol involved coronary angiography at one year post-transplant and every other year thereafter. DSE was used during alternate years following coronary angiography or in lieu of angiography if the recipient creatinine was above $2.0 \mathrm{mg} / \mathrm{dL}$. A diagnostic DSE mandated achievement of $85 \%$ of an age-dependent maximum predicted heart rate (MPHR). All coronary angiograms were graded based on the 2010 ISHLT CAV guidelines ${ }^{2}$. DSE were paired with a coronary angiogram performed within one year of the DSE. If a patient had CAV diagnosed on prior coronary angiography, all subsequent DSE were included in the study irrespective of the duration since angiography.

Corresponding Author: Donna M. Mancini, MD, Center for Advanced Cardiac Care, Department of Medicine, Division of Cardiology, 622 W 168th St, PH 1273, New York, NY 10032, Telephone: 212-305-4629. Fax: 212-305-2591. 
Demographic and clinical variables were summarized with standard descriptive statistics and expressed as mean \pm standard deviation for normally distributed continuous variables, median (with interquartile range) for skewed continuous variables, and count for categorical variables. Group comparison was made with the Chi-squared test.

\section{Results}

A total of 227 DSE were performed on 221 patients. Fifty DSE (22\%) were non-diagnostic: forty-one patients (18\%) did not achieve the MPHR despite receiving the maximal dose of dobutamine (40 $\mu \mathrm{g} / \mathrm{kg} / \mathrm{min}$, atropine was not used) and nine (4\%) were terminated early due to an adverse event (7 hypertension, 1 non-sustained ventricular tachycardia, 1 dizziness). Among the remaining 177 DSE, 154 (87.0\%) had accompanying coronary angiograms, thus constituting the study group.

The study cohort was predominantly male (75.7\%), with a mean age of $58 \pm 13$ years, and median duration since transplant of 2.7 years (interquartile range 2.0-3.1 years). The mean dose of dobutamine used to achieve adequate MPHR and rate-pressure product was $26 \pm 8$ $\mu \mathrm{g} / \mathrm{kg} / \mathrm{min}$ (Table 1). Among the 154 diagnostic DSE with corresponding angiograms, one was positive $(0.6 \%)$, confirmed by coronary angiography as being false positive. Twentynine patients (26.6\%) had CAV on angiography, consistent with the prevalence of CAV three years post-HT in the ISHLT database ${ }^{1}$. There were 28 patients with CAV1 and one with CAV2. Accordingly DSE had a sensitivity of $0 \%(0 / 29)$ to detect early angiographic CAV, a specificity of $99 \%$, a positive predictive value of $0 \%$, and negative predictive value of $81.7 \%$. Survival analysis did not reveal a difference between those with and without CAV; however this was limited by events (one event in each group).

\section{Conclusion}

Dobutamine stress echocardiography is a commonly used technique to screen for CAV in patients following HT. The data presented here, when combined with the recent Cleveland Clinic series ${ }^{5}$ puts into question the external validity of the previous studies from nearly 20 years ago that established the evidence for DSE in CAV screening. This study found that DSE failed to diagnose CAV1 and CAV2 in the first five years following HT.

Screening for CAV is an important component of post-transplant care as it can allow for adjustment in medical therapy to impede further CAV progression. An accurate non-invasive diagnostic technique would be beneficial to all patients, but in particular for those with concomitant renal disease that have a high risk of contrast-induced nephropathy with angiography. A number of alternatives to DSE have been proposed, but all have limitations: CT coronary angiography (radiation, need for contrast, limited accuracy in smaller vessels, need for bradycardia), cardiovascular magnetic resonance imaging (use of gadolinium), positron emission tomography (PET) with coronary flow reserve (CFR) (cost \& radiation), and echocardiography with strain imaging and/or CFR (technical limitations). While evidence to recommend a specific alternative is lacking, PET or echocardiography with measurement of CFR, which measures epicardial and microvascular blood flow, are appealing given the pan-arterial involvement of CAV and the physiological utility. PET with 
CFR has been shown to correlate well with invasive measurements of $\mathrm{CFR}^{6}$ and is prognostic for death, acute coronary syndrome, and heart failure hospitalization ${ }^{7}$. Similarly, in a study of 22 patients, contrast-enhanced transthoracic echocardiography with CFR was found to have good sensitivity $(80 \%)$ and specificity $(100 \%)$ to diagnose $\mathrm{CAV}^{8}$.

This study has two notable limitations. The first is that while paired angiograms and DSE were within a year of each other, they were not concurrent. Second is that the majority (95.4\%) of patients received donor hearts that were younger than the recipient (median 24.5 years, interquartile range 14.8-35.3 years) and the MPHR of the recipient may have been inadequate to induce ischemia in the donor heart.

In conclusion, DSE failed to detect CAV1 and CAV2 in the first five years following HT. Alternative non-invasive means of detecting CAV need to be further investigated to determine the optimal method of non-invasive screening.

\section{References}

1. Lund LH, Edwards LB, Kucheryavaya AY, et al. The Registry of the International Society for Heart and Lung Transplantation: Thirty-second Official Adult Heart Transplantation Report-2015; Focus Theme: Early Graft Failure. J Heart Lung Transplant. 2015; 34:1244-54. [PubMed: 26454738]

2. Mehra MR, Crespo-Leiro MG, Dipchand A, et al. International Society for Heart and Lung Transplantation working formulation of a standardized nomenclature for cardiac allograft vasculopathy-2010. The Journal of Heart and Lung Transplantation. 29:717-27. [PubMed: 20620917]

3. Costanzo MR, Costanzo MR, Dipchand A, et al. The International Society of Heart and Lung Transplantation Guidelines for the care of heart transplant recipients. The Journal of Heart and Lung Transplantation. 29:914-56.

4. Pollack A, Nazif T, Mancini D, Weisz G. Detection and Imaging of Cardiac Allograft Vasculopathy. JACC: Cardiovascular Imaging. 2013; 6:613-23. [PubMed: 23680373]

5. Chirakarnjanakorn S, Starling RC, Popović ZB, Griffin BP, Desai MY. Dobutamine stress echocardiography during follow-up surveillance in heart transplant patients: Diagnostic accuracy and predictors of outcomes. The Journal of Heart and Lung Transplantation. 34:710-7.

6. Wu YW, Chen YH, Wang SS, et al. PET Assessment of Myocardial Perfusion Reserve Inversely Correlates with Intravascular Ultrasound Findings in Angiographically Normal Cardiac Transplant Recipients. Journal of Nuclear Medicine. 2010; 51:906-12. [PubMed: 20484427]

7. Mc Ardle BA, Davies RA, Chen L, et al. Prognostic Value of Rubidium-82 Positron Emission Tomography in Patients After Heart Transplant. Circulation: Cardiovascular Imaging. 2014; 7:930 7. [PubMed: 25182366]

8. Tona F, Osto E, Tarantini G, et al. Coronary Flow Reserve by Transthoracic Echocardiography Predicts Epicardial Intimal Thickening in Cardiac Allograft Vasculopathy. American Journal of Transplantation. 2010; 10:1668-76. [PubMed: 20642688] 
Eligible Population:

221 Patients with 227 DSE

81 Patients Excluded:

Did not have DSE

11 Patients \& 50 DSE Excluded:

Non-diagnostic DSE

21 Patients \& 23 DSE Excluded:

No angiogram within one year

Study Cohort:

109 Individual Patients with a total of 154 DSE

Figure 1.

Patient selection 


\section{Table 1}

Patient and Study Characteristics

\begin{tabular}{|ll|}
\hline Age $(\mathrm{y})$ & $57.8 \pm 13.5$ \\
Male $(\%)$ & 75.7 \\
Time from HT (y) & $2.7 \pm 1.0$ \\
Max Dobutamine Dose $(\mu \mathrm{g} / \mathrm{kg} / \mathrm{min})$ & $26.4 \pm 8.1$ \\
Age MPHR (bpm) & $87.1 \pm 2.2$ \\
Rate-pressure product (RPP) & $21,251 \pm 4,387$ \\
Rest-Stress RPP Difference & $9,093 \pm 4,262$ \\
CAV & \\
$\quad$ Grade 1 & 28 \\
$\quad$ Grade 2 & 1 \\
\hline
\end{tabular}

Continuous variables presented as mean \pm standard deviation. MPHR=Maximum predicted heart rate, CAV=Cardiac Allograft Vasculopathy, HT=Heart Transplantation 